九州大学学術情報リポジトリ

Kyushu University Institutional Repository

\title{
Transition from a punched-out dislocation to a slip dislocation revealed by electron tomography
}

Tanaka, Masaki

Department of Materials Science and Engineering, Kyushu University

Liu, Grace S.

Department of Materials Science and Engineering, University of Illinois

Kishida, Tomonobu

Mitsubishi Heavy Industries, Ltd. | Department of Materials Science and Engineering, Kyushu University

Higashida, Kenji

Department of Materials Science and Engineering, Kyushu University

他

http://hdl. handle. net/2324/26347

出版情報: Journal of Materials Research. 25 (12)，pp.2292-2296，2010-12. Cambridge University Press

バージョン：

権利関係: (C) 2010 Materials Research Society 


\title{
Transition from a punched-out dislocation to a slip dislocation revealed by electron tomography
}

\author{
Masaki Tanaka ${ }^{\text {a) }}$ \\ Department of Materials Science and Engineering, Kyushu University Nishi-ku, \\ Fukuoka 819-0395, Japan \\ Grace S. Liu \\ Department of Materials Science and Engineering, University of Illinois, Urbana, Illinois 61801 \\ Tomonobu Kishida ${ }^{\text {b) }}$ and Kenji Higashida \\ Department of Materials Science and Engineering, Kyushu University Nishi-ku, \\ Fukuoka 819-0395, Japan \\ Ian M. Robertson ${ }^{\text {c) }}$ \\ Department of Materials Science and Engineering, University of Illinois, Urbana, Illinois 61801
}

(Received 29 July 2010; accepted 7 September 2010)

\begin{abstract}
Punched-out dislocations emitted from an octahedral oxide precipitate in single-crystal silicon were investigated using high-voltage electron microscopy and tomography (HVEM-tomography) to understand the mechanism of softening caused by the oxide precipitates. In the present paper, direct evidence of the transition of a punched-out prismatic dislocation loop to a slip dislocation is presented. The punched-out dislocation grows into a large matrix dislocation loop by absorption of interstitial atoms, which were produced during oxide precipitation.
\end{abstract}

\section{INTRODUCTION}

Oxide precipitates are included in some silicon wafers to increase the purity of the active device area, with the concomitant enhancement of the electrical properties. ${ }^{1}$ Either octahedral or platelet precipitates can be grown by performing the appropriate heat treatments. ${ }^{2}$ Metallic ions are trapped at lattice defects produced around the precipitates. These defects may take the form of punched-out prismatic dislocation loops or stacking faults. It has been reported that the precipitates affect not only the optical properties but also the mechanical properties. ${ }^{3-9}$ For example, the yield stress of silicon with precipitates can be up to $33 \%$ lower than that of silicon with no precipitates. ${ }^{5}$ The magnitude of the decrease is dependent on the precipitate size. One of the proposed mechanisms to explain the decrease in yield stress involves the punchedout dislocations transitioning to Frank-Read sources. ${ }^{4}$ However, punched-out dislocations cannot themselves be Frank-Read sources as the segment length of punched-out dislocations is usually less than a few hundred nanometers

\footnotetext{
a) Address all correspondence to this author. e-mail: tanaka.masaki.760@m.kyushu-u.ac.jp

b) Present address: Mitsubishi Heavy Industries, Ltd., 10 Ooe-cho, Minato-ku, Nagoya, 455-8515 Japan.

c) This author was an editor of this journal during the review and decision stage. For the JMR policy on review and publication of manuscripts authored by editors, please refer to http://www.mrs. org/jmr_policy
}

DOI: $10.1557 / J M R .2010 .0308$ so that the resolved shear stress to bow out the segment is too large. In the present paper, direct evidence of the transition from a punched-out dislocation to a slip dislocation is presented; electron tomography ${ }^{10-12}$ was used to reveal the dislocation distribution around a precipitate three-dimensionally.

\section{EXPERIMENTAL}

A (001) P-type Czochralski silicon wafer containing octahedral precipitates with an average size of $300 \mathrm{~nm}$ and a density of $4 \times 10^{10} \mathrm{~cm}^{-3}$ were used. Three-point bend specimens of dimensions $8 \times 35 \times 0.63 \mathrm{~mm}^{3}$ were cut from the wafer. A V-shaped notch was introduced at the tensile edge of the specimen, and three-point bend tests were performed at $993 \mathrm{~K}$ to an applied load of $66 \mathrm{~N}$. The notch tip area was thinned to electron transparency using a Fischione-1010 ion mill, with the final specimen thickness being approximately $2 \mu \mathrm{m}$. The TEM observation was carried out using a high-voltage electron microscope (HVEM; JEM-1300NEF) operating at an accelerating voltage of $1250 \mathrm{kV}$. A side-entry double-tilt specimen holder was used, the maximum tilt angle of which is $\pm 40^{\circ}$ around the longitudinal direction of the holder and $\pm 45^{\circ}$ around the perpendicular direction. The specimen was continuously tilted in the specimen holder, and the images needed were acquired every $2^{\circ}$ to reconstruct a three-dimensional tomogram. The doubletilt specimen holder enables keeping the diffraction condition nearly constant during the acquisition of the 
images. No subsequent image processing or filtering was performed, as the inherent contrast from each image was sufficient for alignment using the Inspect3D software. Then a three-dimensional reconstruction, or tomogram, of the volume was produced from the aligned images using EM3D software, which was then used for producing a traced 3D model of the volume.

\section{RESULTS AND DISCUSSION}

Figure 1(a) shows a bright-field (BF) HVEM image of an oxide precipitate before the deformation. With the incident beam direction and foil normal both [001], the precipitates exhibit a regular octahedral shape as shown schematically in Fig. 1(b). The crystal structure of the precipitate was reported as amorphous $\mathrm{SiO}_{2}{ }^{13}$ with the habit plane along $\{111\}_{\mathrm{Si}}$. No punched-out dislocation loops were observed around the precipitate at this stage.

Figure 2 shows a BF image of a precipitate after deformation at $993 \mathrm{~K}$. The incident beam direction was again

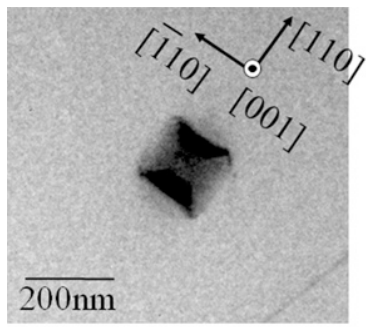

(a)

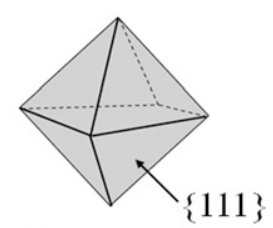

(b)
FIG. 1. (a) Bright-field image of a precipitate in a silicon single crystal. The foil normal and incident beam directions are both [001]. The diffraction vector is $\mathbf{g}=220$. (b) The precipitate, which has a habit plane of $\{111\}_{\mathrm{Si}}$.

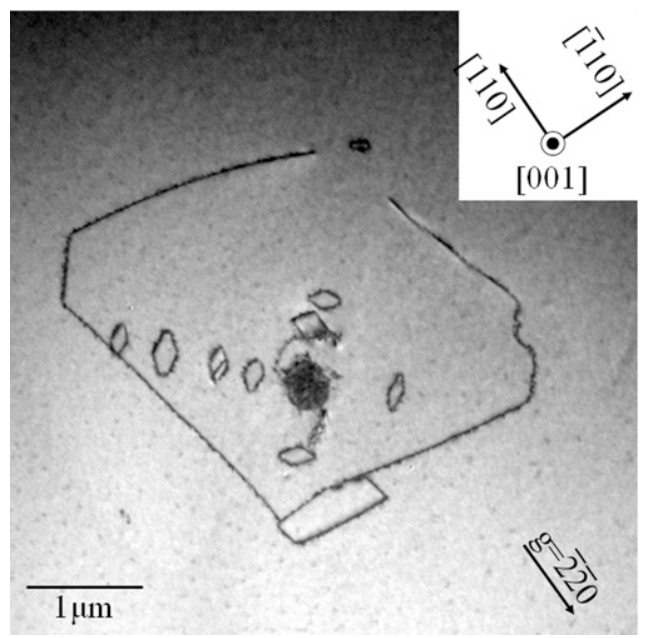

FIG. 2. Bright-field image of punched-out dislocations and a large dislocation loop around a precipitate. The foil normal and incident beam directions are [001]. The precipitate was damaged by the irradiated electron beam during image series acquisition. parallel to [001]. The black circle contrast at the center of the figure marks the prior position of the precipitate, which was damaged in the process of observation. The black dot contrast in the matrix shows small dislocation loops, presumably interstitial in nature, produced by the electron beam; the threshold displacement voltage is 200 to $400 \mathrm{kV}$ in silicon, which is below the operating voltage of the microscope. These loops however, are readily distinguishable from the loops produced by the deformation and do not impact the current analysis.

Three kinds of dislocation loops were observed: eight regular punched-out dislocation loops, one elongated punched-out dislocation loop, and one large dislocation loop surrounding the punched-out dislocations. The octahedral precipitate was located at the center of the large dislocation loop. The two dislocation segments next to the precipitate, the Burgers vector of which was determined to be $a / 2[110]$, are likely the remnants of a recently punched-out loop that intersected the foil surface during sample thinning. The specimen was tilted approximately $\pm 40^{\circ}$ with respect to 220 and images acquired approximately every $2^{\circ}$. The diffraction condition was kept constant $\mathbf{g}=\overline{2} \overline{2} 0$ during the acquisition of the tilt series. A spatially and tilt-aligned animation of these BF images is shown in supplementary movie file 1 , and the reconstructed image is shown in supplementary movie file 2. In addition to acquiring these images, other diffraction conditions were used to permit determination of the Burgers vector of the dislocations by using the $\mathbf{g} \cdot \mathbf{b}$ invisibility criterion.

Figure 3 shows images from the BF tilt series, the reconstructed tomogram, and a $3 \mathrm{D}$ model traced from the tomogram. The specimen was tilted $-38^{\circ}$ in Fig. 3(a), $0^{\circ}$ in Fig. 3(b), and $35^{\circ}$ in Fig. 3(c). The views in Figs. 3(d), 3(e), 3(f), and 3(g), 3(h), 3(i) correspond to the BF images in Figs. 3(a), 3(b), and 3(c), respectively. The tilt angle indicates the deviation between incident beam direction and the [001] foil normal direction. The punched-out dislocation labeled $A$ in Fig. 3(a), viewed edge-on, appears to be absent in Fig. 3(b) - this is a projection effect as this dislocation loop overlaps another punched-out dislocation, as indicated by the arrow in Fig. 3(b). A similar observation could be made for the loop labeled $B$ : while present in Figs. 3(a) and 3(b), it appears to be absent in Fig. 3(c); this is due to the superposition of the dislocation on the precipitate. A slight difference in the imaging condition also influences the contrast of certain segments of dislocations, such as the weaker contrast of $B$ in Fig. 3(a) compared with that in Fig. 3(b), or the fainter appearance of a segment of the large dislocation loop in Fig. 3(c). These instances illustrate the dangers of interpretation based on only a few micrographs, as information in the beam direction cannot be resolved directly. We see, however, that this ambiguity is easily dispelled by viewing the aligned tilt series 


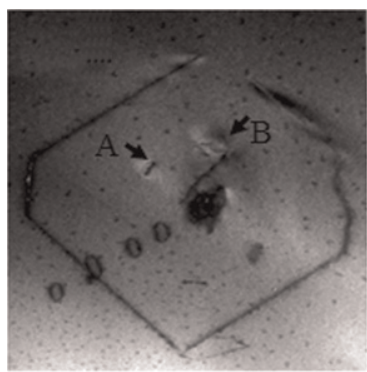

(a)

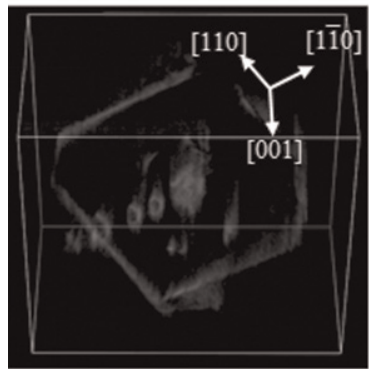

(d)

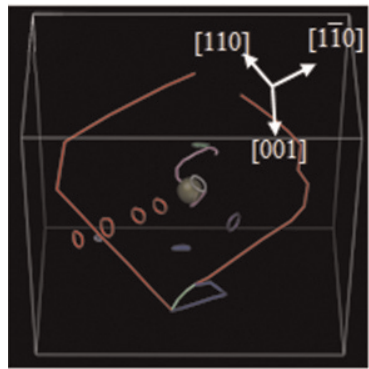

(g)

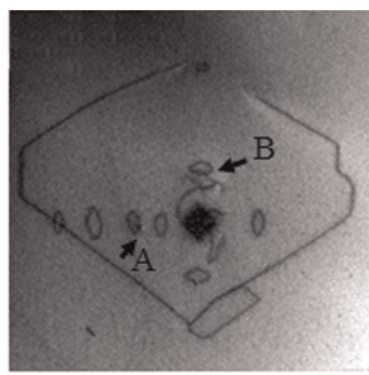

(b)

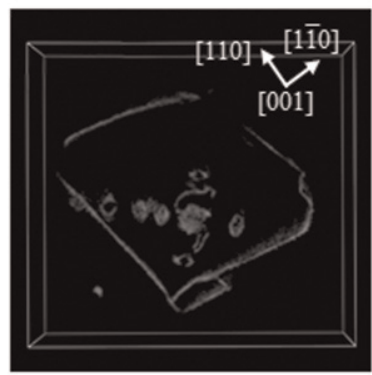

(e)

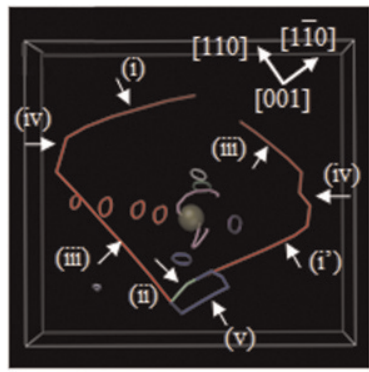

(h)

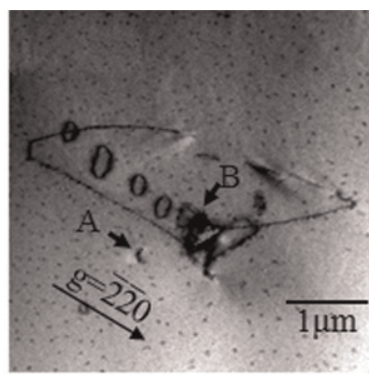

(c)

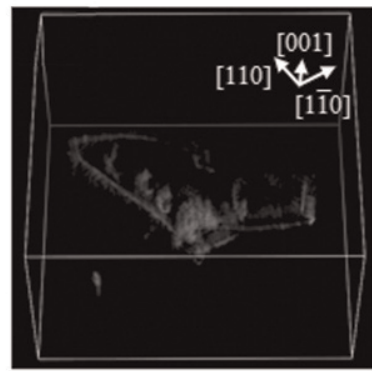

(f)

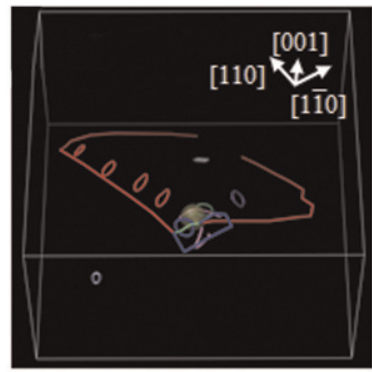

(i)

FIG. 3. Punched-out dislocations around a $\mathrm{SiO}_{2}$ particle. (a)-(c) Bright-field TEM images from the tilt series; and (d)-(f) corresponding views from the reconstructed tomogram, and (g)-(i) the $3 \mathrm{D}$ traced model based on the tomogram. The specimen thickness is approximately $2 \mu \mathrm{m}$. (color online)

acquired over a large angular range, where the position of each dislocation is well distinguished throughout the series. The aligned tilt series is presented in supplementary movie file 1 . Although the diffraction condition is maintained, the diffraction condition locally changes around one segment in the big loop at a high tilt angle, which makes one segment of the big loop faint in Fig. $3(\mathrm{c})$. The tilt series also reveals the crystallographic plane of the large dislocation loop. The dislocation segments labeled (i), (i'), and (ii) are on the ( $\overline{1} \overline{1} 1)$ slip plane; those labeled (iii) are on the (1111); those labeled (iv) lie along the $[2 \overline{1} \overline{1}]$ direction on the (111), but have a Burgers vector of $a / 2[011]$, which is not on the (111) slip plane. Therefore segments labeled (iv) are sessile, as the line direction and Burgers vector define the slip plane as (011) and not the accepted (111). In a previous study, ${ }^{9}$ all segments of this kind of dislocation were considered to be on the identical $\{111\}$ slip plane, which left the origin of the large dislocation loop ambiguous. It is also still ambiguous whether the dislocation loop can be a new dislocation source or not. HVEM tomography reveals that segments of the large dislocation all reside on different planes, and the loop becomes a dislocation source, which is discussed next.

In Fig. 3, each dislocation segment is colored according to its Burgers vector. The Burgers vector of the large dislocation loop is $a / 2[011]$, which is the same as that of the four punched-out dislocations, stringing toward the left in Fig. 3. One of the segments labeled (ii) in the large dislocation loop interacts with a part of the punched-out dislocations colored blue labeled (v). The Burgers vector of the interacted segment (ii), colored light green, changed from $a / 2[011]$ to $a / 2[110]$ after interacting with a segment of the elongated punchedout dislocation, blue, which has a Burgers vector of $a / 2$ [10i] ]. This reaction is as expected for interacting dislocations. With this Burgers vector, dislocation segment (ii) is also sessile. Note that both ends of dislocation segment $\left(\mathrm{i}^{\prime}\right)$ are anchored by sessile dislocations, thus allowing segment $\left(i^{\prime}\right)$ to act as a Frank-Read source. 
Here, the Frank-Read stress, $\tau_{\mathrm{FR}}$, for a segment of the punched-out dislocation and that of the large loop can be estimated. The stress needed to activate a Frank-Read source is determined at the condition when the loop radius is equal to half the distance between the pinning segments, ${ }^{14,15}$ and is

$$
\tau_{\mathrm{FR}}=\frac{\mu \mathbf{b}}{\lambda},
$$

where the shear modulus $\mu$ is $0.681 \times 10^{11} \mathrm{~Pa}$, the magnitude of the Burgers vector of the dislocation $\mathbf{b}$ is $0.384 \times 10^{-9} \mathrm{~m}$, and $\lambda$ is the length of the dislocation segment $\left(\mathrm{i}^{\prime}\right)$. For the segment in the punched-out dislocation and $\left(\mathrm{i}^{\prime}\right)$ in the large loop, $\lambda$ is approximately 0.3 and $3.5 \mu \mathrm{m}$, which correspond to a Frank-Read stress of 90 and $7 \mathrm{MPa}$, respectively. The latter stress level is low enough for the dislocation segment to operate as a Frank-Read source. It means that a punched-out dislocation cannot operate as a Frank-Read source initially, but must grow to a sufficient size before it is capable of acting as one. The Burgers vector of the segments colored red and the slip planes of segments labeled (i), ( $\left.i^{\prime}\right)$, (iii) and (iv) are the same as those of the punched-out dislocations colored red. This suggests that the large dislocation loop grew from one of the punched-out dislocations. Since the dislocation segments labeled (iv) are out of the slip plane, the large loop grew by dislocation climb. The temperature for the bending test in this study is $993 \mathrm{~K}$, which is $0.59 T_{\mathrm{m}}$, where $T_{\mathrm{m}}$ is the melting temperature. The test temperature is sufficient for dislocation climb to occur. Dislocation cross slip has been reported to occur near a stress concentrator such as a crack tip at $973 \mathrm{~K},{ }^{16}$ although cross slip was not observed often in this study. The expansion of a dislocation loop via climb is discussed next.

To accommodate the compressive stress field around a precipitate, extrinsic prismatic dislocation loops that consist of an extra half-plane in the loop interior must be emitted. Since the punched-out dislocations are extrinsic prismatic loops ${ }^{17}$ excess interstitial atoms are necessary to enable them to grow. Since the punched-out dislocation is small compared with the large loop, the total number of interstitial atoms inside the large loop is determined by dividing the area by the square of the Burgers vector. The area surrounded by the dislocation loop is $7.5 \times 10^{-12} \mathrm{~m}^{2}$; thus, the number of atoms enclosed, $N_{\mathrm{I}}$, is:

$$
N_{\mathrm{I}}=7.5 \times 10^{-12} / \mathrm{b}^{2}=5.1 \times 10^{7} \text { atoms } .
$$

The reaction formula for a single silica oxide deep inside silicon is generally given by the following equation with the assumption that vacancies are not operative $e^{1,18}$

$$
2 \mathrm{Si}+2 \mathrm{O}_{\mathrm{i}}=\mathrm{SiO}_{2}+\mathrm{I}_{\mathrm{Si}} .
$$

In Eq. (3), $\mathrm{O}_{\mathrm{i}}$ and $\mathrm{I}_{\mathrm{Si}}$ denote interstitial oxygen and silicon, respectively. The formula indicates that interstitial silicon atoms are emitted around the precipitate when an oxide precipitate is generated in the specimen. The number of interstitial atoms emitted from the volume of the precipitates is given by

$$
n_{\mathrm{Si}}=n_{\mathrm{SiO}_{2}}=\frac{\rho \cdot V}{M_{\mathrm{SiO}_{2}}},
$$

where $n_{\mathrm{SiO}_{2}}$ is the number of $\mathrm{SiO}_{2}$ molecules, $\rho=2.33 \times$ $10^{6}$ is the mass density of $\mathrm{SiO}_{2}, V$ is the volume of $\mathrm{SiO}_{2}$, and $M_{\mathrm{SiO}_{2}}$ is the molecular mass. Taking the diameter of the octahedral precipitate in Fig. 2 to be approximately $300 \mathrm{~nm}$, this gives $V=4.5 \times 10^{-21} \mathrm{~m}^{3}$, which gives $n_{\mathrm{SiO}_{2}}=1.1 \times 10^{8}$ atoms. Thus, the number of interstitial atoms generated during the oxide precipitation is sufficient for a punched-out dislocation to grow to the size of the large dislocation loop by climb.

\section{CONCLUSIONS}

The three-dimensional distribution of the dislocation structures around an octahedral precipitate was determined using HVEM tomography. It was found that: (i) a large dislocation loop develops around the precipitate. (ii) The Burgers vector of the large dislocation loop is the same as that of one of the punched-out dislocations, which indicates that the large dislocation loop is most likely formed by the growth of the punched-out dislocations through climb. (iii) One of the dislocation segments in the large dislocation loop is anchored by sessile segments, which enable the large dislocation loop to act as a Frank-Read source. (iv) The calculated number of interstitial atoms emitted during the precipitation is large enough to explain the growth of the punched-out dislocation. (v) The growth of the punched-out dislocations leads to an increase of dislocation sources in silicon single crystals, which leads to macroscopic softening of the silicon matrix.

\section{ACKNOWLEDGMENTS}

M.T. and K.H. acknowledge that this work is partly supported by Grant-in-Aid for Scientific Research (KAKENHI Nos. 22246090 and 22760544). G.S.L. and I.M.R. acknowledge support from the U.S. Department of Energy through Grant No. DE-FE02-07ER46443.

\section{REFERENCES}

1. A. Borghesi, B. Pivac, A. Sassella, and A. Stella: Oxygen precipitation in silicon. J.Appl. Phvs. 77, 4169 (1995).

2. K. Sueoka, N. Ikeda, T. Yamamoto, and S. Kabayashi: Morphology change of oxide precipitates in $\mathrm{CZ}$ silicon during 2-step annealing. J.Electrochem. Soc. 141, 3588 (1994).

3. K. Jurkschat, S. Senkader, and P.R. Wilshaw: Onset of slip in silicon containing oxide precipitates. J. Appl. Phvs. 90, 3219 (2001). 
4. A. Giannattasio, S. Senkader, R.J. Falster, and P.R. Wilshaw: The role of prismatic dislocation loops in the generation of glide dislocations in $\mathrm{Cz}$-silicon. Comput. Mater. Sci. 30, 131 (2004).

5. I. Yonenaga and K. Sumino: Mechanical behavior of Czochralskisilicon crystals as affected by precipitation and dissolution of oxygen atoms. Jpn. J. Appl. Phvs. 21, 47 (1982).

6. B. Leroy and C. Plougonven: Warpage of silicon wafers. J. Electrochem. Soc. 127, 961 (1980).

7. K. Yashutake, M. Umeno, and H. Kawabe: Mechanical properties of heat-treated Czochralski-grown silicon crystals. Appl. Phys. Lett. 37, 787 (1980)

8. R. Behrensmeier, M. Brede, and P. Haasen: The influence of precipitated oxygen on the brittle-ductile transition of silicon. $\underline{\mathrm{Scr}}$. Metall. 21. 1581 (1987).

9. K. Sueoka, M. Akatsuka, and H. Katahama: Dependence of mechanical strength of Czochralski silicon wafers on temperature of oxygen precipitation annealing. J. Electrochem. Soc. 144, 1111 (1997).

10. J.S. Barnard, J. Sharp, J.R. Tong, and P.A. Midgley: High-resolution three-dimensional imaging of dislocations. Science 313, 319 (2006)

11. J.H. Sharp, J.S. Barnard, K. Kaneko, K. Higashida, and P.A. Midgley: Dislocation tomography made easy: A reconstruction from ADF STEM images obtained using automated image shift correction. J.Phvs. Conf. Ser. 126, 012013 (2008).
12. M. Tanaka, K. Higashida, K. Kaneko, S. Hata, and M. Mitsuhara: Crack tip dislocations revealed by electron tomography in silicon single crystal. Scr. Mater. 59, 901 (2008).

13. F.A. Ponce and S. Hahn: Structure of thermally-induced microdefects in Czochralski silicon, in Electron Microscopy of Materials, edited by W.A. Krakow, D.A. Smith, and L.W. Hobbs (Mater. Res. Soc. Symp. Proc. 31, North-Holland, New York, 1984), p. 153.

14. J.P. Hirth and J. Lothe: Theory of Dislocations (McGraw-Hill, New York, 1986).

15. G.E. Dieter: Mechanical Metallurgy, 2nd ed. (McGraw-Hill Book Company, New York, 1976).

16. M. Tanaka, S. Sadamatsu, G.L. Liu, H. Nakamura, K. Hgashida, and I.M. Robertson: Sequential multiplication of dislocation sources along a crack front revealed by HVEM-tomography. J. Mater. Res. (submitted).

17. T.Y. Tan and W.K. Tice: Oxygen precipitation and the generation of dislocations in silicon. Philos. Mag. 34, 615 (1976).

18. T.Y. Tan: Exigent-accommodation volume of precipitation and formation of oxygen precipitates in silicon, in Oxygen, Carbon, Hydrogen and Nitrogen in Crystalline Silicon, edited by J.C. Mikkelsen, Jr., S.J. Pearton, J.W. Corbett, and S.J. Pennycook (Mater. Res. Soc. Symp. Proc. 59, Pittsburgh, PA, 1986), p. 269. 